\title{
Single pyrimidine discrimination during voltage-driven translocation of osmylated oligodeoxynucleotides via the a-hemolysin nanopore
}

\author{
Yun Ding ${ }^{1}$ and Anastassia Kanavarioti ${ }^{*}$
}

\author{
Full Research Paper \\ Address: \\ ${ }^{1}$ Chemistry Department, University of Utah, Salt Lake City, UT, USA \\ and ${ }^{2}$ Yenos Analytical LLC, EI Dorado Hills, CA, USA \\ Email: \\ Anastassia Kanavarioti* - tessi.kanavarioti@gmail.com \\ * Corresponding author \\ Keywords: \\ DNA sequencing; $\alpha$-hemolysin; ion-channel measurements; \\ nanopore; osmium tetroxide bipyridine; osmylated oligos; osmylation; \\ single-stranded DNA (ssDNA)
}

Beilstein J. Nanotechnol. 2016, 7, 91-101.

doi:10.3762/bjnano.7.11

Received: 28 August 2015

Accepted: 08 January 2016

Published: 22 January 2016

Associate Editor: N. Motta

(c) 2016 Ding and Kanavarioti; licensee Beilstein-Institut. License and terms: see end of document.

\begin{abstract}
The influence of an electric field on an isolated channel or nanopore separating two compartments filled with electrolytes produces a constant ion flux through the pore. Nucleic acids added to one compartment traverse the pore, and modulate the current in a sequence-dependent manner. While translocation is faster than detection, the $\alpha$-hemolysin nanopore ( $\alpha$-HL) successfully senses base modifications in ssDNA immobilized within the pore. With the assistance of a processing enzyme to slow down translocation, nanopore-based DNA sequencing is now a commercially available platform. However, accurate base calling is challenging because $\alpha$-HL senses a sequence, and not a single nucleotide. Osmylated DNA was recently proposed as a surrogate for nanopore-based sequencing. Osmylation is the addition of osmium tetroxide 2,2'-bipyridine (OsBp) to the C5-C6 pyrimidine double bond. The process is simple, selective for deoxythymidine (dT) over deoxycytidine (dC), unreactive towards the purines, practically $100 \%$ effective, and strikingly independent of length, sequence, and composition. Translocation of an oligodeoxynucleotide (oligo) $\mathrm{dA}_{10} \mathrm{XdA}_{9}$ via $\alpha-\mathrm{HL}$ is relatively slow, and exhibits distinct duration as well as distinct residual current when $\mathrm{X}=\mathrm{dA}, \mathrm{dT}(\mathrm{OsBp})$, or $\mathrm{dC}(\mathrm{OsBp})$. The data indicate that the $\alpha$-HL constriction zone/ $\beta$-barrel interacts strongly with both OsBp and the base. A 23 nucleotide long oligo with four $\mathrm{dT}(\mathrm{OsBp})$ traverses 18-times slower, and the same oligo with nine $(\mathrm{dT}+\mathrm{dC})(\mathrm{OsBp})$ moieties traverses 84 -times slower compared to $\mathrm{dA}_{20}$, suggesting an average rate of 40 or $180 \mu \mathrm{s} / \mathrm{base}$, respectively. These translocation speeds are well above detection limits, may be further optimized, and clear the way for nanopore-based sequencing using osmylated DNA.
\end{abstract}

\section{Introduction}

Nanopore technology has seen great advancements following a rather slow start as a global analytical tool for anything from cells to single molecules $[1,2]$. Progress in X-ray crystallogra- phy to deduce structural details of protein pores [3-5], and progress in manufacturing of nanometer-sized holes in thin layers of inorganic materials [6-9] has led to exploration of both 
natural and synthetic pores for a number of applications $[10,11]$. The concept, reintroduced independently by David Deamer and George Church in the context of DNA sequencing, is based on applying a potential across an open pore embedded in an insulating surface that separates two compartments filled with electrolyte. A nucleic acid in one compartment can move through the pore to the other compartment influenced by the electric field and the interactions with the pore, and concurrently modulate the current. Protein pores such as the $\alpha$-HL, a modified version of the Mycobacterium smegmatis porin A (MspA), and the Ph29 connector channel have been investigated as single molecule sensing devices for ssDNA, RNA, dsDNA, and proteins [12-14]. The advantage of the natural pores is that they are well defined and highly reproducible, whereas the advantage of the solid-state nanopores is that they can be manufactured to desirable dimensions. Sensing rare DNA bases produced by UV-radiation and other oxidative processes was successfully accomplished by chemically modifying the base and/or immobilizing the strand within the $\alpha$-HL pore [15-17]. Such measurements revealed that current obstruction upon passing of a telomere DNA via the pore yields folding information [18]. The observation that $\alpha$-HL can distinguish between RNA and DNA homopolymers [19-21] led to a large effort in government, academia, and industry to explore nanopores for DNA sequencing [22-27].

Early on it became evident that translocation of ssDNA, at about 1 to $2 \mu$ s per base, is too fast to report back on the small current modulation associated with the passing of each base [13]. It also became clear that the protein pores, such as $\alpha-\mathrm{HL}$ and MspA, have sensing regions that interact with a sequence and not a single base $[13,28-30]$. Attempts to overcome these challenges include modification of the wild type proteins by site-directed mutagenesis or other means to improve/narrow the sensing region [30-32], tagging of the bases by amino acids or by PEGylation [33-35], assessing the specific current level sensed by the pore for each possible sequence in order to create a complete data set of current signatures [36], and the use of improved bioinformatic tools $[37,38]$. Incorporation of a processing enzyme, phi29 DNA polymerase, at the top of the pore to move the nucleic acid one base at a time, was a game changer because it slowed down translocation to the ms level [39-41]. Translocation speed in the presence of an enzyme is reduced by three to four orders of magnitude and it is two to three orders slower than necessary for detection, yielding a tentative 100 to 1000 -fold reduction in reading speed compared to an optimal situation [12,13]. In a nutshell progress in nanopore-based sequencing of DNA is still limited by three issues: (i) the chemical comparability of the four nucleobases in the context of current modulation, (ii) the fact that translocation in the absence of a processing enzyme is too fast for detection and in the presence of such enzyme too slow for reading genomes, and (iii) that so far no system detects and reports back a distinct signal that corresponds to a single base.

Minimal effort has been placed so far in labeling one or more of the bases with a moiety to add bulkiness and impose discrimination, perhaps, because selective labels are hard to find and because proof of efficient labeling of a gigabase long nucleic acid is seen as an unattainable goal. While working with metalorganic molecules to label ssDNA, we evaluated OsBp [42]. OsBp is known to add to the C5-C6 double bond of the pyrimidine ring (Scheme 1). Because osmium is a good contrast agent for imaging by electron microscopy (EM), osmylated DNA was proposed 60 years ago and exploited in attempts to obtain DNA sequence information by EM imaging [43-45]. The

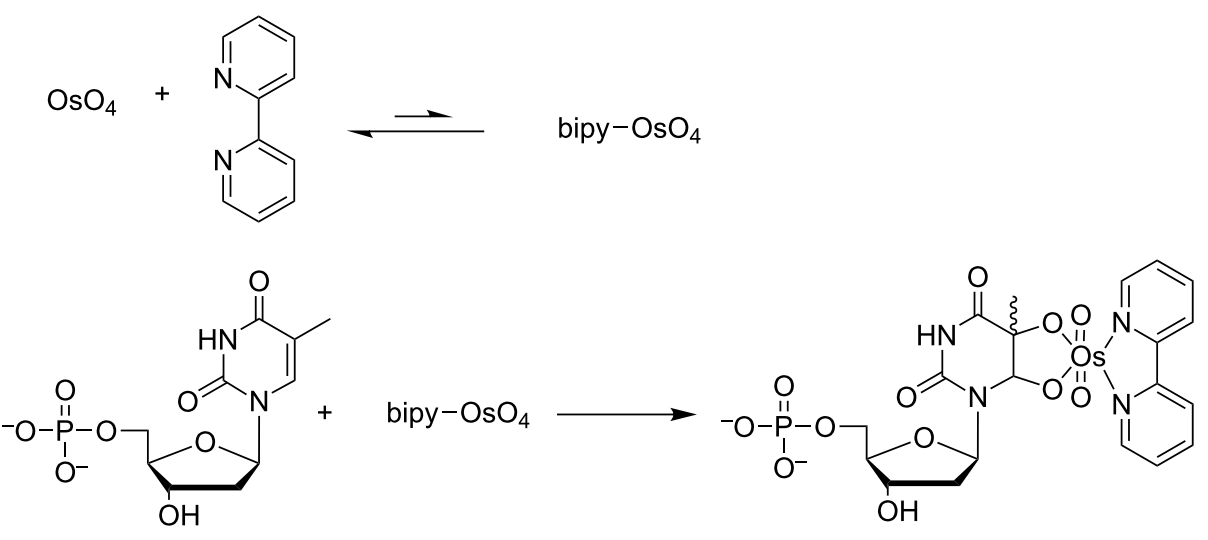

Scheme 1: OsBp with dTMP. Reaction of osmium tetroxide with 2,2'-bipyridine forms a reactive complex (OsBp or bipy-OsO $\left.{ }_{4}\right)$, which in a second step reacts with a pyrimidine (thymidine monophosphate (dTMP) shown here) to form the osmylated pyrimidine $[42,44]$. One way to illustrate the difference between osmylated and intact bases is to compare (molecular weight) of each: dC (111), dT (126), dA (135), dG (151); dC-OsBp (521), dT-OsBp (536), i.e., osmylation adds about $400 \%$ mass to the reactive base compared to the unreactive one. 
more recent advancement of nanopores as single molecule detection devices and the corresponding progress in manufacturing, parallelization, and commercialization of such platforms [24], supported the idea of testing osmylated DNA as a surrogate for nanopore-based sequencing (see Figure 1 and [46,47]).

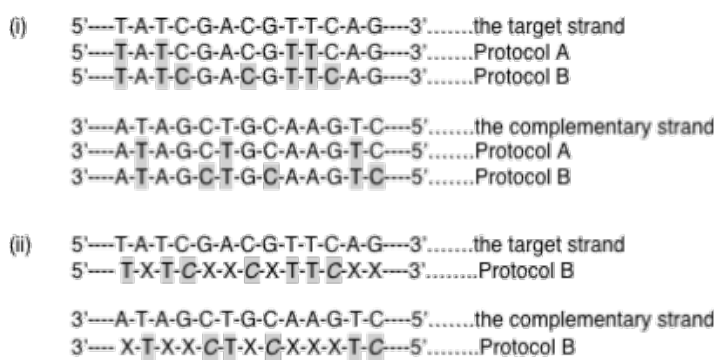

Figure 1: Strategy for sequencing DNA using the osmylated strands All sequences shown refer to deoxybases; for simplicity $d$ is left out. (i) Original proposed route for obtaining the sequence of the target strand by osmylating both the target strand and its complementary with Protocols A and B [46]; Protocol A yields primarily osmylated-T (shaded) and Protocol B yields practically $100 \%$ osmylated pyrimidines (shaded $\mathrm{T}$ and $\mathrm{C}$ ). Sequencing the complementary strand provides indirectly information on A and G. This approach presumes discrimination between osmylated from intact base, but no discrimination among pyrimidines. (ii) The data presented in this report strongly suggest that $\alpha-\mathrm{HL}$ discriminates osmylated-T (shaded regular font) from osmylated-C (shaded italic font) and intact purine $(X)$. Therefore a streamlined strategy might be to sequence osmylated strands using only one protocol, perhaps Protocol B. For further discussion on the sequencing strategy [47].

The proposition was motivated by studies showing that the osmylation [48] is a remarkably clean reaction yielding the ex- pected products in practically $100 \%$ yield with no detectable side-reactions. An example is provided in Figure 2 where the reaction between $\mathrm{OsBp}$ and $\mathrm{dA}_{10} \mathrm{dCdA}_{9}$ is monitored by capillary electrophoresis (CE) analysis. Any side-reaction, or any backbone degradation, or oxidative damage would have been detectable as additional peaks with typical detectability of $0.1 \%$. Any product formed by these processes is expected to retain some nucleotides, and therefore be spectrophotometrically visible. Indeed the only detectable reaction is the conversion of $\mathrm{dA}_{10} \mathrm{dCdA}_{9}$ to $\mathrm{dA}_{10} \mathrm{dC}(\mathrm{OsBp}) \mathrm{dA}$. Evidence for the addition of one unit OsBp per pyrimidine double bond has been reviewed in the literature [43-45] and was specifically tested under our conditions by UV-vis, and ${ }^{1} \mathrm{H}$ NMR [42]. Moreover molecular masses corresponding to adducts with 1, 2, or 3 OsBp moieties were obtained by MALDI TOF for oligos with 1, 2, or $3 \mathrm{Ts}$, respectively [42].

Unpublished data suggest false positives and false negatives to be below $1 / 10,000$, a remarkable feat for any modification reaction. The reactivity is not impeded by long sequences of pyrimidines, as evidenced by the rate for complete osmylation of $\mathrm{dT}_{15}$ that is, within experimental error, comparable to the rate of monomer osmylation, i.e., dTTP to $\operatorname{dTTP}(\mathrm{OsBp})$ [46]. It turns out that the same protocol, in the absence of any denaturing agents, works predictably and reproducibly for short and long oligos, as well as for M13mp18, a circular 7459 bases long ssDNA with secondary structure [46]. The success in using the same protocol for ssDNA with secondary structure as for short oligos is attributed to the hydrophobicity of the OsBp moiety

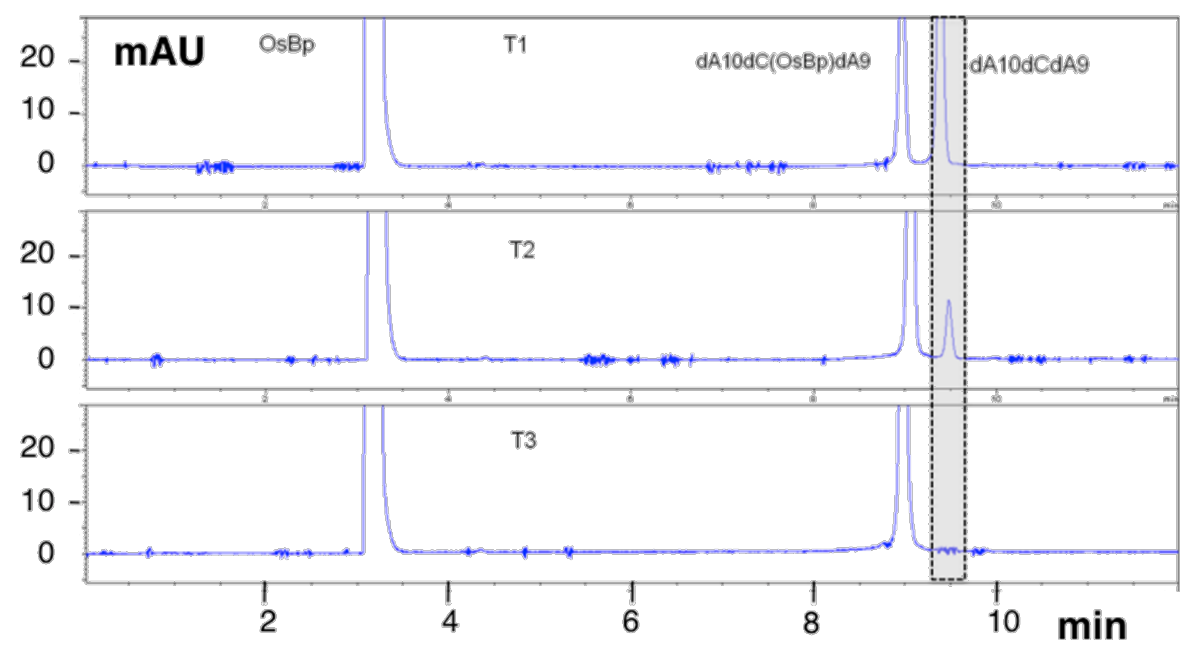

Figure 2: Reaction of $12.6 \mathrm{mM}$ OsBp (the highest concentration of OsBp used under our conditions) with $20 \mu \mathrm{M} \mathrm{dA} \mathrm{A}_{10} \mathrm{dCdAg}$ monitored automatically by CE (see Experimental section, Part A). Reactants were mixed in a CE vial and aliquoted/analyzed automatically by the instrument. T1 represents $6 \mathrm{~min}$, T2 $24 \mathrm{~min}$ and T3 $76 \mathrm{~min}$ incubation after mixing. Signal is shown at $260 \mathrm{~nm}$. Reaction mixture was incubated at $25 \pm 1{ }^{\circ} \mathrm{C}$ within the thermostated autosampler of the instrument. Shaded peak is the original oligo that migrates last, OsBp is neutral and migrates early. The reaction product (osmylated oligo) migrates ahead of the intact oligo. Oligo $\mathrm{dA}_{10} \mathrm{dCdA}_{9}$ used in this reaction was PAGE-purified with confirmed purity by CE at $97 \%$. The small spikes, at about $1 \mathrm{mAU}$ with no steady migration time, are due to noise; the peak height of both oligo and osmylated oligo reach $120 \mathrm{mAU}$. 
that disrupts base stacking interactions [49]. This feature implies that any ssDNA of unknown sequence can be predictably osmylated. Additionally osmylated oligos and osmylated ssDNA are stable at room temperature for days as shown by CE analysis both in the presence of OsBp, or after purification/removal of the excess label $[42,46]$.

It was also shown that the osmylation product exhibits a new chromophore in the range 300 to $320 \mathrm{~nm}[42,46]$ where DNA does not absorb (see below in Figure 3). This chromophore was the basis for developing a UV-vis assay to quantitatively measure the extent of osmylation, and facilitate the development of the two protocols (Figure 1(i)); Protocol A exploits low concentration of OsBp with short incubation, and yields primarily dT(OsBp), and Protocol B uses higher concentration $(12.6 \mathrm{mM})$ with longer incubation and yields practically $100 \%(\mathrm{dT}+\mathrm{dC})(\mathrm{OsBp})$; both protocols work at room temperature. The UV-vis assay serves as a quality control assay to confirm extent of osmylation.

The realization that osmylation adds a 4-fold mass to the reacting base (Scheme 1, caption) fueled the speculation that any size-suitable nanopore could discriminate between osmylated and native base, and led to a proposed sequencing strategy (Figure 1). Preliminary experiments to assess pore size suitability using solid-state silicon nitride nanopores [50] showed that $1.6 \mathrm{~nm}$ wide pores permit translocation of 80 -mer long osmylated oligos, and exhibit dramatic translocation slowdown with enhanced osmylation. These observations led us to undertake the $\alpha$-HL nanopore measurements reported here.

\section{Results and Discussion Interaction of $\alpha-H L$ nanopore with single osmylated pyrimidine during oligo translocation}

This is the first study to assess translocation properties of osmylated oligos via the $\alpha$-HL nanopore. Based on chemical struc- ture the expectation was that the constriction site of $\alpha-\mathrm{HL}$ at $1.4 \mathrm{~nm}$ might be too narrow to allow translocation of osmylated oligos. Nevertheless experiments with solid-state nanopores at $1.6 \mathrm{~nm}$ diameter provided evidence for translocation [50], and so do the experiments with $\alpha$-HL described below. Here we explored the translocation properties of a series of 20-mer oligoadenylates where the 11th nucleotide was a deoxypyrimidine, such as $\mathrm{dT}, \mathrm{dC}, 5-\mathrm{Me}-\mathrm{dC}$, and $\mathrm{dU}$, as well as a 23-mer oligo consisting of all four bases. To explore the effect on oligo entry in the $\alpha$-HL pore we also tested the later with a $\mathrm{dA}_{25}$ tail added either to the 3 '- or the 5'-end (Table 1).

The oligos used in this study were purchased from Integrated DNA Technologies, as PAGE-purified and desalted materials (Table 1). They were analyzed by capillary electrophoresis (CE) to assess purity, and then osmylated (see Experimental section); R1 refers to Protocol A and yields primarily dT(OsBp) and R2 refers to Protocol $\mathrm{B}$ and yields $(\mathrm{dT}+\mathrm{dC})(\mathrm{OsBp})$ oligos. The products were purified from excess OsBp in a spin-minicolumn, and analyzed by $\mathrm{CE}$ to confirm the extent of osmylation (see below Table 2, 2nd column, and Figure 3), as well as the absence of unreacted OsBp. The modified oligos were evaluated via the $\alpha$-HL pore using an instrument custom made for the Chemistry Department of the University of Utah equipped with a glass pore membrane (GNM, see Experimental section). Ion channel measurements were done using conditions for unmodified oligos as described in [14]: $10 \mu \mathrm{M}$ oligo in $1.00 \mathrm{M} \mathrm{KCl}$, at pH 7.4 with $10 \mathrm{mM}$ potassium phosphate buffer at $22 \pm 1{ }^{\circ} \mathrm{C}$. Up to four different probing voltages, namely 100, 120, 140, and $160 \mathrm{mV}$ (trans vs cis) were tested.

The observation of decreasing dwell times with increasing voltage (Table 2) is strong evidence that the osmylated oligo translocated. Five $20 \mathrm{nt}$ long oligodeoxyadenylates were screened: $\mathrm{dA}_{20}$ serving as control, and four $\mathrm{dA}_{10} \mathrm{XdA}$, where $\mathrm{X}$ is an osmylated pyrimidine: $X=\mathrm{dT}(\mathrm{OsBp}), \mathrm{dC}(\mathrm{OsBp})$, 5-MedC(OsBp), or $\mathrm{dU}(\mathrm{OsBp})$. Translocation time for $\mathrm{dA}_{20}$ was obtained from the maximum value of a Gaussian curve analysis

\begin{tabular}{|c|c|c|c|}
\hline ODN & $\%$ purity by $\mathrm{CE}$ & $N_{\text {total }}$ & sequence : deoxyoligonucleotide (5'-3') \\
\hline $\mathrm{dA}_{20}$ & 83 & 20 & AAA AAA AAA AAA AAA AAA AA \\
\hline $\mathrm{dA}_{10} \mathrm{dTd} \mathrm{A}_{9}$ & 86 & 20 & AAA AAA AAA ATA AAA AAA AA \\
\hline $\mathrm{dA}_{10} \mathrm{dCd}_{9}$ & 86 & 20 & AAA AAA AAA ACA AAA AAA AA \\
\hline $\mathrm{dA}_{10} 5-\mathrm{MedCd} \mathrm{A}_{9}$ & 74 & 20 & AAA AAA AAA A5-MeCA AAA AAA AA \\
\hline $\mathrm{dA}_{10} \mathrm{dUd} \mathrm{A}_{9}$ & 78 & 20 & AAA AAA AAA AdUA AAA AAA AA \\
\hline pGEX3' & 89 & 23 & CCG GGA GCT GCA TGT GTC AGA GG \\
\hline $\mathrm{dA}_{25}$-pGEX3' & 88 & 48 & $(A)_{25}$ CCG GGA GCT GCA TGT GTC AGA GG \\
\hline pGEX3'-dA 25 & 95 & 48 & CCG GGA GCT GCA TGT GTC AGA GG $(\mathrm{A})_{25}$ \\
\hline
\end{tabular}




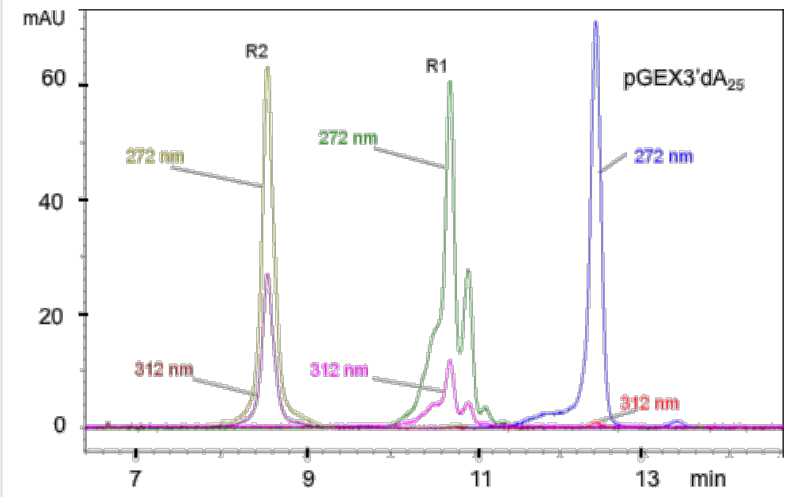

Figure 3: Capillary electrophoresis (CE) overlapping traces of oligodeoxynucleotides pGEX3'-dA 25 intact and pGEX3'-dA 25 at R1 and R2 levels of osmylation per protocols $A$ and $B$, respectively (Experimental section, Part A). Materials are at comparable, but not identical, concentrations. Migration is in the order of intact oligo last, R2 early, and $\mathrm{R} 1$ in the middle. Traces are shown at two wavelengths, at 272 and $312 \mathrm{~nm}$, to illustrate that DNA exhibits about $1 \%$ absorbance, whereas $\mathrm{R} 1$ and R2 absorb substantially, and R2 > R1. The detail in the R1 peak is attributed to different topoisomers produced from either top or bottom addition to the C5-C6 double bond. Topoisomers exist also with R2, but are too many to be resolved. The ratio $R(312 \mathrm{~nm} / 272 \mathrm{~nm})$ represents a normalization, and the wavelengths are selected to maximize the value of $R$ [46].

of the data and values at different voltages were as expected for this set up (Table 2, first entry). Translocation data from the other oligos were fitted with a first-order exponential decay, and provided translocation times, $\tau$, also illustrated in Figure 4 and listed in Table 2. Typically current levels $\left(I_{0}\right)$ in unobstructed pores are about $120 \pm 5 \mathrm{pA}$ at $120 \mathrm{mV}$ (conductance $\approx 1000 \mathrm{pS}$ ) at our conditions. Normalized values of residual current $\left(I_{\mathrm{r}} / I_{0}\right)$ were obtained from the maximum value of the current histograms and are also reported in Table 2; they are accurate to $\pm 1 \%$, and did not vary with voltage.

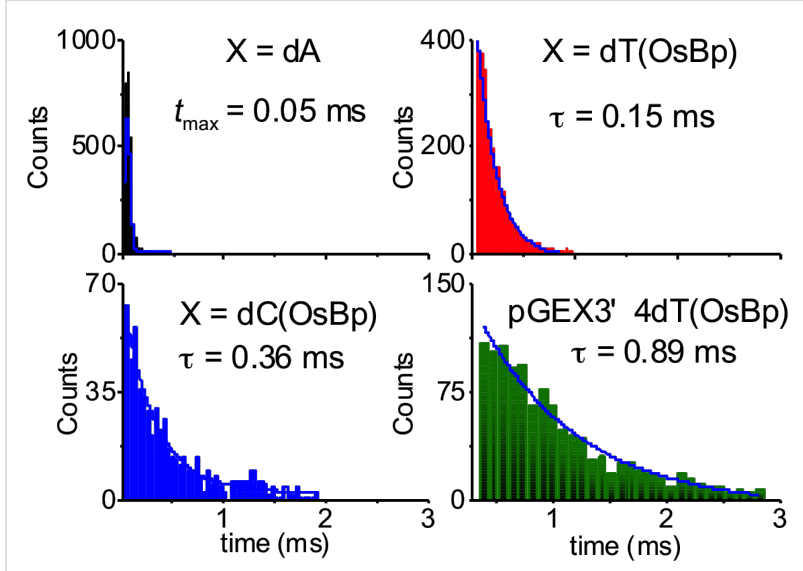

Figure 4: Translocation time histograms for four different oligos: Three are oligodeoxyadenylates, $\mathrm{dA}_{10} \mathrm{XdA_{9 }}$ where $\mathrm{X}=\mathrm{dA}, \mathrm{dT}(\mathrm{OsBp})$, or $\mathrm{dC}(\mathrm{OsBp})$. The fourth is a 23 nt long deoxyoligo pGEX3' (PCR primer) and contains four modified bases, dT(OsBp). Translocation speed decreases in the presence of a single modified pyrimidine; $\mathrm{dC}$ slows down the oligo more compared to $\mathrm{dT}$, and the presence of additional osmylated bases yields further slow-down.

Evidence for translocation of osmylated oligos via the $\alpha$-HL pore, despite the apparent bulkiness of the OsBp moiety, was initially surprising. Actually the approximately orthogonal positioning of the nucleobases to the strand axis, and the top or bottom addition of OsBp to the pyrimidine double bond, lead to the conjecture that OsBp stretches parallel to the strand direction (Figure 5) and not perpendicular to it, which would enlarge the perimeter of this modified DNA. As speculated [46] the

\begin{tabular}{|c|c|c|c|c|c|c|c|}
\hline \multirow[t]{2}{*}{ material } & \multirow[t]{2}{*}{$\mathrm{R} 1$ or $\mathrm{R} 2$} & \multirow[t]{2}{*}{ number of osmylated bases } & \multirow[t]{2}{*}{$I_{\mathrm{r}} / I_{0}( \pm 1 \%)$ at $120 \mathrm{mV}$} & \multicolumn{4}{|c|}{ translocation time, $\mathrm{T}(\mathrm{ms})$} \\
\hline & & & & $100 \mathrm{mV}$ & $120 \mathrm{mV}$ & $140 \mathrm{mV}$ & $160 \mathrm{mV}$ \\
\hline $\mathrm{dA}_{20}$ & 0.01 & 0 & 0.14 & 0.07 & 0.05 & 0.03 & a \\
\hline $\mathrm{dA}_{10} \mathrm{dTd} \mathrm{A}_{9}$ & 0.11 & $1(\mathrm{~T})$ & 0.08 & 0.20 & 0.15 & 0.10 & - \\
\hline $\mathrm{dA}_{10} \mathrm{dCd} \mathrm{A}_{9}$ & 0.11 & $1(\mathrm{C})$ & 0.11 & - & 0.36 & 0.26 & 0.24 \\
\hline $\mathrm{dA}_{10} 5-\mathrm{MedCdA}$ & 0.11 & $1(5-\mathrm{MeC})$ & 0.12 & - & 0.31 & 0.24 & 0.20 \\
\hline $\mathrm{dA}_{10} \mathrm{dUd} \mathrm{A}_{9}$ & 0.11 & $1(\mathrm{dU})$ & 0.12 & - & 0.47 & 0.36 & 0.30 \\
\hline pGEX3', R1 & 0.42 & $4(\mathrm{~T})$ & 0.06 & $-^{b}$ & 0.89 & 0.49 & 0.35 \\
\hline pGEX3', R2 & 0.77 & $9(T+C)$ & 0.03 & 6.45 & 4.20 & 3.50 & - \\
\hline $\mathrm{dA}_{25}$-pGEX3', R1 & 0.20 & $4(T)$ & 0.10 & $-^{b}$ & 0.65 & 0.45 & 0.36 \\
\hline pGEX3'-dA $25, R 1$ & 0.20 & $4(\mathrm{~T})$ & 0.11 & $-^{b}$ & 0.37 & 0.28 & 0.17 \\
\hline pGEX3'-dA $25, R 2$ & 0.43 & $9(T+C)$ & 0.07 & $-^{b}$ & $0.53^{c}$ & $0.39^{c}$ & $0.17^{\mathrm{c}}$ \\
\hline
\end{tabular}

aToo fast to be analyzed with a $100 \mathrm{kHz}$ filter and $500 \mathrm{kHz}$ acquisition rate; bevent frequency is too low to allow population analysis in a reasonable amount of time; 'cthere are three different populations, the number here shows the dwell time for the first population ( $<10 \mathrm{~ms})$. 
presence of the OsBp moiety reduced the residual current and slowed down the translocation of the modified oligo. However the markedly different ion-channel measurements observed between $\mathrm{dA}_{10} \mathrm{dT}(\mathrm{OsBp}) \mathrm{dA}_{9}$ and $\mathrm{dA}_{10} \mathrm{dC}(\mathrm{OsBp}) \mathrm{dA}_{9}$ were unexpected (see Graphical Abstract, Figure 4, and Table 2). It turns out that $\mathrm{dT}(\mathrm{OsBp})$ yields more relative current obstruction, but faster translocation compared to $\mathrm{dC}(\mathrm{OsBp})$. The observation that electrophoretic properties associated with a single modification in an oligo are so different is unprecedented, and strongly suggests interaction between the pore, most likely the constriction site of $\alpha-\mathrm{HL}$, with both the OsBp moiety and the nucleobase. It is not clear whether or not those interactions are direct or indirect via the corresponding solvation shells. It is plausible that the presence of OsBp inside the constriction site provides a highly confined environment in which the modified nucleotide is forced to rearrange to a less favored configuration as well as to a different solvation shell that "carries along" only what is critically important; all these changes are then detected as current modulation and slower dwell time.

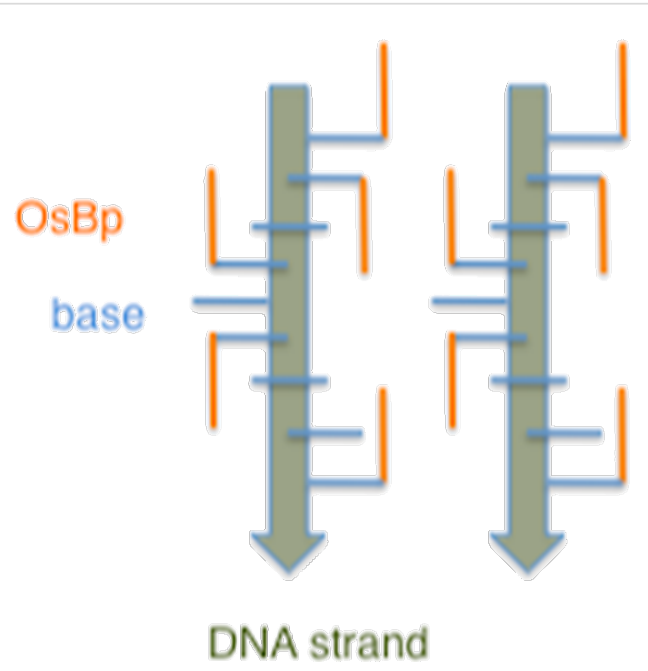

Figure 5: Representation of osmylated DNA strands to illustrate the practically parallel line-up of the OsBp moieties to the direction of the strand. This picture also demonstrates the plausible complexation of two strands by hydrophobic interactions between the OsBp moieties. Note that OsBp can add from the top or the bottom of the C5-C6 double bond, and vary from molecule to molecule (not shown here). Complexation is favored in solutions at relatively high oligo and high salt concentration.

In an attempt to understand the source of the differences between $\mathrm{dT}(\mathrm{OsBp})$ and $\mathrm{dC}(\mathrm{OsBp})$ we included $\mathrm{dA}_{10} 5$ MedC(OsBp)dA 9 and $\mathrm{dA}_{10} \mathrm{dU}(\mathrm{OsBp}) \mathrm{dA}_{9}$, expecting that 5 -MedC would perhaps mimic dT and dU would mimic $\mathrm{dC}$. Even though current modulation is comparable among 5-MedC, $\mathrm{dC}$, and $\mathrm{dU}$, dwell times are substantially different and in the order $\mathrm{dU}>\mathrm{dC}>5-\mathrm{MedC}>\mathrm{dT}>>\mathrm{dA}$ with $\tau=0.47,0.36,0.31$, 0.15 and $0.05 \mathrm{~ms}$ at $120 \mathrm{mV}$ (trans vs cis), respectively. These differences are well above experimental error, they are observed at all three different voltages, i.e., at 120, 140 and $160 \mathrm{mV}$ (see Table 2), and clearly suggest a forceful interaction between the base and the nanopore for all four osmylated bases. As seen in Table 2 the residual current is 2 to $3 \%$ more blocking for osmylated $\mathrm{dC}, 5-\mathrm{MedC}$, and $\mathrm{dU}$, and $6 \%$ more blocking for osmylated dT compared to intact dA. These differences appear small when compared to differences observed for immobilized bases within the pore [16]. It is noteworthy that in the present experiments the measured current modulation is "averaged" over the full sequence of the oligo. Hence it is reasonable to expect that discrimination at the single base level of an immobilized osmylated oligo would be larger than what is observed here, and it might not lead to overlap as seen in the current histogram on the TOC graphic.

\section{Dramatic translocation slow-down of osmy- lated oligos}

At $120 \mathrm{mV}$ the translocation speed of the oligoadenylate is reduced by a factor of 3 , when the middle nucleotide (nt) is replaced by dT-OsBp (duration increased from 0.05 to $0.15 \mathrm{~ms}$ ), and by a factor of 7 , when it is replaced by $\mathrm{dC}(\mathrm{OsBp})$ (duration increased from 0.05 to $0.36 \mathrm{~ms}$ ). To explore how osmylation affects translocation of a typical oligo, we tested pGEX3', a $23 \mathrm{nt}$ PCR primer, as well as two $48 \mathrm{nt}$ long oligos with the sequence of pGEX3' and an added $\mathrm{dA}_{25}$ tail either at the 3 '-end or at the 5'-end (see Table 1 and Table 2). The osmylated pGEX3' (R1, 4 dT(OsBp)) exhibits 18-fold or 16-fold slower translocation at 120 or $140 \mathrm{mV}$, respectively, compared to the control $\mathrm{dA}_{20}$ (durations increased from 0.05 to $0.89 \mathrm{~ms}$ at $120 \mathrm{mV}$ (Figure 4) and from 0.03 to $0.49 \mathrm{~ms}$ at $140 \mathrm{mV}$ ). A $0.89 \mathrm{~ms}$ translocation for a $23 \mathrm{nt}$ oligo yields an average speed of $40 \mu$ s per base, a measurable quantity by current state-of-the art instruments, indicating that the use of a processing enzyme to show down DNA translocation could be avoided. Complete osmylation of both pyrimidines in pGEX3' R2 (with $4 \mathrm{dT}(\mathrm{OsBp})+5 \mathrm{dC}(\mathrm{OsBp})$ ) yields further current obstruction, $11 \%$ more blocking, compared to $\mathrm{dA}_{20}$ and $3 \%$ more blocking compared to pGEX3' R1. Most likely the low current levels observed with pGEX3' $\mathrm{R} 2$ arise from the sequence region where seven out of eleven nucleotides are osmylated pyrimidines. These data suggest that translocation is sensitive to extent of osmylation, an observation made also with SiN nanopores [50]. The dwell times observed with pGEX3' R2 are dramatically slow and decrease with increasing voltage. The process at $120 \mathrm{mV}$ is 84 -times slower compared to $\mathrm{dA}_{20}$ and yields $180 \mu \mathrm{s}$ per base speed for an oligo with a $39 \%$ pyrimidine content.

Notably, the event frequency decreases sharply with osmylation and part of it can be attributed to the slower translocation. Number of events per second are $19,8,4$, and 0.2 for $\mathrm{dA}_{20}$ with 
no OsBp, dA $10 \mathrm{dT}(\mathrm{OsBp}) \mathrm{A}_{9}$ with 1 OsBp, pGEX3' R1 with 4 OsBp and pGEX3' R2 with 9 OsBp moieties, respectively. In particular the decrease in events frequency between four osmylated dTs and nine osmylated pyrimidines hints to an issue that could be rationalized by complexation of two oligos as follows: Considering that OsBp is a highly hydrophobic moiety, two oligos could associate via hydrophobic interactions along the OsBp moieties (see Figure 5), coexist in the wide vestibule of the $\alpha$-HL pore, and need to dissociate first before, at least one, translocates. Such phenomenon would sharply reduce the probability to traverse, and even create artifacts like the second population of events observed with the two R2 oligos (see below in Figure 6). It is plausible that this hydrophobic association is favored in the $10 \mu \mathrm{M}$ oligo concentration and in the presence of $1 \mathrm{M} \mathrm{KCl}$ used for the present experiments. Lower oligo and/or lower salt concentrations may suppress association, yield higher frequency of events, a single and more narrowly defined population, and a better understanding of R2 translocation features.

Another way to rationalize the extreme slow-down was to assume the presence of an unidentified impurity in the OsBp preparation that, at the higher concentrations used for Protocol $\mathrm{B}$, yields substantial amounts of cross-linking between strands to form a duplex covalently bound, in a parallel manner, like an " $\mathrm{H}$ ". We tested this hypothesis by monitoring the OsBp labeling reaction with a $32 \mathrm{nt}$ long deoxyoligo, pGEX3'-dA9 (tail at the 3 '-end), by high performance liquid chromatography (HPLC) with ion exchange chromatography (see Experimental section). The oligo was chosen so that cross-linking of two strands to form a $64 \mathrm{nt}$ long conjugate could be easily detected by this HPLC method. The presence of the tail is not expected to have a major effect on the reactivity of the alleged OsBp impurity. Even though we monitored the reaction 3-times longer than protocol B required, there was no detectable formation of longer oligos, with an upper limit of $0.05 \%$, consistent with absence of such impurity.

In an attempt to facilitate translocation and enhance event frequency, we investigated two 48-mers, based on the pGEX3' sequence, one bearing an $\mathrm{dA}_{25}$ tail at the 5'-end and the other bearing the $\mathrm{dA}_{25}$ tail at the 3 '-end (Table 1 and Table 2). Comparison of the 48-mers at the R1 level with the pGEX3' R1 indicates that the two longer oligos exhibit comparable current obstruction levels, but obstruct current less compared to the 23 -mer. In addition, the dwell times of both 48 -mers at $120 \mathrm{mV}$ are shorter compared to the parent oligo (see heat plots in Figure 3). Still the dwell times at 140 and $160 \mathrm{mV}$ are comparable between the 23-mer and the 48-mer with the tail at the 5 '-end suggesting that at the higher voltages the tail has no effect on the electrophoretic behavior that is practically deter- mined by the modified sequence. Even though current obstruction is comparable for the two R1 level 48-mers, there is a marked difference in dwell times across all three voltages showing that the 48 -mer with the tail at the 3 '-end moves almost twice the speed of the 48-mer with the tail at the 5 '-end. This behavior is consistent with preference for 3'-entry established experimentally and computationally for native ssDNA [51]. In contrast to the other oligos that exhibit one population in event plots, the R2 level oligos, pGEX3' R2 and pGEX3'$\mathrm{dA}_{25} \mathrm{R} 2$, exhibit two and three, respectively (Figure 6; the third population in $\mathrm{pGEX}^{\prime}$ '- $\mathrm{dA}_{25} \mathrm{R} 2$ lies outside the graph in the time range). The observation of more than one populations hints to issues with the hydrophobicity of the OsBp moiety discussed above and perhaps with non-optimal experimental conditions. As expected the residual current for these two R2 oligos are lower compared to the corresponding currents observed with the R1 oligos and in the order pGEX3' R2 < pGEX3'-dA 25 R2, pointing out that the $\mathrm{dA}_{25}$ tail assists in translocation.
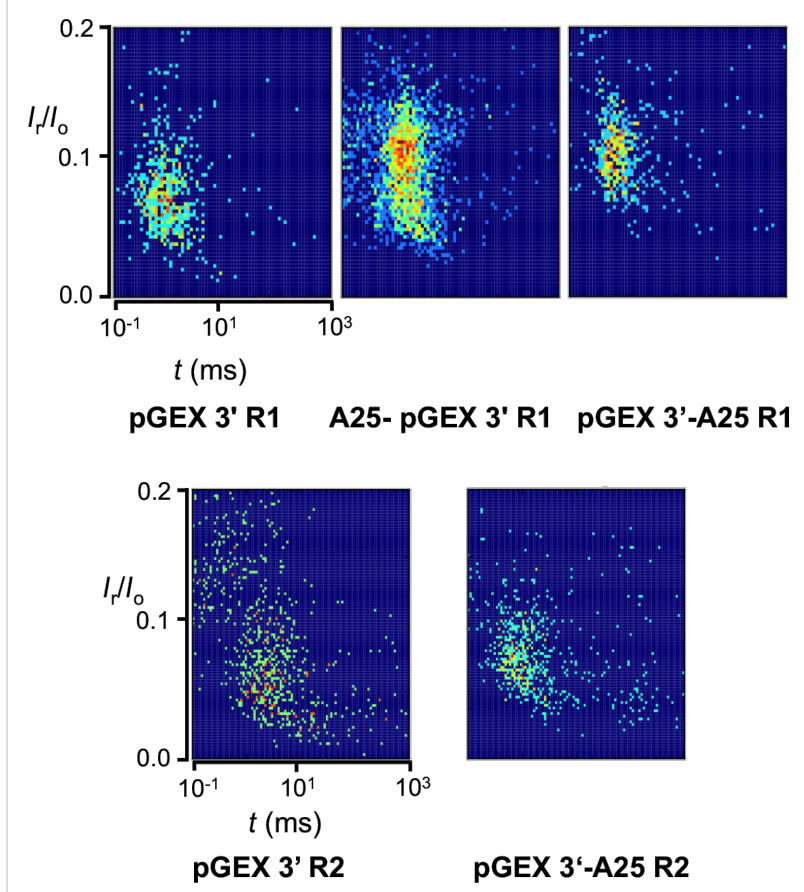

Figure 6: Heat plots, normalized residual current, $I_{\mathrm{r}} / I_{0}$, vs translocation time, $t(\mathrm{~ms})$, for the deoxyoligos with multiple osmylated pyrimidines. pGEX3' is a $23 \mathrm{nt}$ long PCR primer; R1 stands for $4 \mathrm{dT}(\mathrm{OsBp})$ and $\mathrm{R} 2$ stands for $\{4 \mathrm{dT}(\mathrm{OsBp})+5 \mathrm{dC}(\mathrm{OsBp})\}$. Event plots with $\mathrm{R} 1 \mathrm{ex}-$ hibit a single population. Event plots with R2 exhibit, at least, two populations consistent with other mechanisms operating besides single molecule translocation. pGEX3'- $A_{25}$ R2 exhibits a third population (not shown) at times longer than $1 \mathrm{~s}$. In all cases the $A_{25}$-tail facilitates translocation and more so when it is at the 3 '-end compared to the 5'-end.

Inspection of the $I-t$ traces for the pGEX3'-type osmylated oligos revealed many different patterns. Events for $\mathrm{dA}_{25^{-}}$ 
pGEX3' R1 (4 OsBp) exhibited 0, 1, or 2 blockage interruptions or "steps", see Figure 7. This phenomenon can be rationalized with the help of Figure 5 and Figure 7. Due to its size (estimated at about $0.8 \mathrm{~nm}$ ) the OsBp moiety extends from the base, that it is attached to, all the way to the third base in the sequence and provides partial "coverage" to two internucleotide spaces (see red blocks in Figure 7). Depending on the direction of the osmylation (orange arrows, top or bottom), the presence of osmylated Ts in a sequence can easily lead to an apparent uninterrupted blockage, as shown schematically in sequence a of Figure 7, blockage interrupted once (sequence b), or blockage interrupted twice (sequence c). Even though the planar structure of OsBp may extend along the strand, it cannot encircle the adjacent base. Hence we speculate that if the overall current blockage was not as severe as it is under our conditions, the intact bases might have made their presence detectable by influencing the current. Optimization of the conditions to produce more current, or change to a wider/shorter pore, such as a solid-state nanopore, may yield better discrimination between an osmylated base and the adjacent intact one. Even using the present conditions one should be able to show experimentally $\mathrm{X}$ number of steps in $I-t$ events for an oligo composed of $\mathrm{X}+1$ pyrimidines separated by at least five purines.

\section{Conclusion}

In summary, osmylated oligos traverse the $\alpha$-HL nanopore and block measurably more current compared to the unmodified control. Translocation speeds with osmylated oligos are slow, decrease with degree of osmylation, and reach detectable levels by current state-of-the-art instruments. The observation that the nanopore discriminates by both residual current and dwell time, among a single $\mathrm{dT}(\mathrm{OsBp})$, a single $\mathrm{dC}(\mathrm{OsBp})$, and a purine, provide proof-of-principle for nanopore-based sequencing using osmylated DNA as a surrogate. Further optimization of the conditions is necessary to suppress hydrophobic complexation between strands and improve discrimination. Obtaining sequence information directly from dsDNA is envisioned as follows: In the presence of a denaturant, dsDNA may be subject to osmylation by protocol B and yield two osmylated ssDNA strands. The osmylated target strand could provide sequence information for $\mathrm{T}, \mathrm{C}$, and purine, and the osmylated complementary strand sequence information for A, G, and pyrimidine (bases in the target). Mathematically speaking the system is fully described. Moreover the number of consecutive pyrimidines in the target strand at any location in the sequence should be identical to the number of consecutive purines in the corresponding location of the complementary strand. The last feature gives a handle to partially compensate for top or bottom addition of OsBp to base. The results reported here make the strategy outlined in Figure 1 (ii) testable.

\section{Experimental}

Part A, Materials, oligos, preparation of osmylated oligos, capillary electophoresis (CE) analysis, and high performance liquid chromatography (HPLC) analysis: HPCE grade solution of $50 \mathrm{mM}$ sodium tetraborate $\mathrm{pH} 9.3$ was purchased from Agilent Technologies. A 4\% aqueous osmium tetroxide solution $(0.1575 \mathrm{M}$ in ampules at $2 \mathrm{~mL}$ each) was purchased from Electron Microscopy Sciences. 2,2'-Bipyridine $99+\%$ (bipyridine) was purchased from Acros Organics. Oligos were purchased from Integrated DNA Technologies (IDT), diluted with DNase/RNase-free water (from MP Biomedical) to $2 \mu \mathrm{g} / \mathrm{mL}$ or $100 \mu \mathrm{M}$ and stored at $-20^{\circ} \mathrm{C}$. The purity of these oligos was tested using $\mathrm{CE}$ in $50 \mathrm{mM}$ sodium tetraborate at

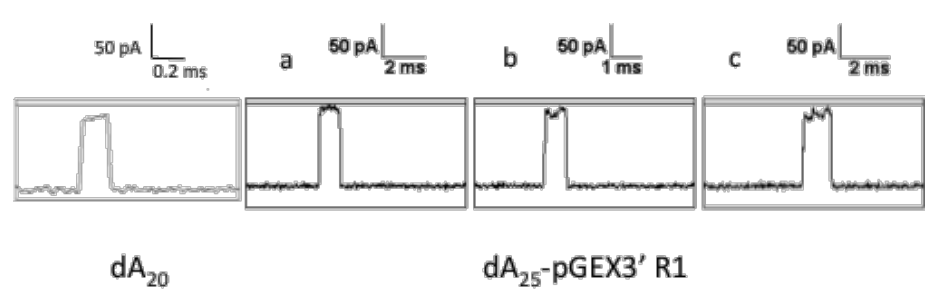

(a) (A) ${ }_{25}$-CCGGGAGqIGCATGIGICAGAGG

(b) (A) 25 -CCGGGAGGIGCATGTG

(c) (A) ${ }_{25}$-CCGGGAGCTGCATJGTICĀ'GAGG

Figure 7: Sample $1-t$ traces for the control $\mathrm{dA}_{20}$ and for $\mathrm{dA}_{25}-$ pGEX3' $\mathrm{R} 1$ (with $4 \mathrm{~T}(\mathrm{OsBp})$ ) to show (a) continuous blockage, (b) blockage interrupted once, or (c) blockage interrupted twice. These inter-events steps may be attributed to selected configurations (a to c) as above. Theoretically three interruptions of blockages are expected for 4 modifications. Arrows indicate OsBp moiety and have direction; red blocks indicate partial coverage of adjacent bases due to the presence of OsBp. The planar structure of OsBp prohibits full coverage of adjacent bases (not shown in the 2D configuration above). Note that there is more than one plausible configuration to rationalize type $b$ and type $c$ events. 
pH 9.3 with $20 \mathrm{kV}$. Oligos used in this study, their sequences and purity are listed in Table 1 . The oligo used for the experiment in Figure 2, was a different batch of much better purity compared to the one listed in Table 1.

Analyses were carried out using an Agilent $1600 \mathrm{CE}$ instrument equipped with diode array detector (DAD) and Chemstation software, Rev.B.04.02SP1, for data acquisition and processing. Untreated fused silica capillaries $(50 \mathrm{~mm} \times 40 \mathrm{~cm})$ with extended light path were purchased from Agilent Technologies. Monitoring of reactions was conducted using the same CE method as for purity check (see above). For identification purposes we abbreviate R1 (312/272) the ratio of the absorbance at the two wavelengths for the product of the reaction following Protocol A, and R2 (312/272) the corresponding ratio following protocol B (see below). The values R1 and R2 serve as quality control of the product because they can be calculated from the relationships $\mathrm{R} 1=2.21 \times \mathrm{dT} / N_{\text {total }}$ and $\mathrm{R} 2=2.01 \times(\mathrm{dT}+\mathrm{dC}) / N \mathrm{t}_{\mathrm{otal}}$, where $N_{\text {total }}$ is the total number of nucleotides, $\mathrm{dT} / N_{\text {total }}$ is the fraction of $\mathrm{dT}$, and $(\mathrm{dT}+\mathrm{dC}) / N_{\text {total }}$ is the fraction of pyrimidines in the sequence [46]. Unpublished kinetic determinations under identical conditions reveal intrinsic selectivity for osmylation of $\mathrm{dT} / \mathrm{dC}=28$ (in agreement with previous data [42]), $\mathrm{dT} / 5-\mathrm{MedC}=6.9,5-\mathrm{MedC} / \mathrm{dC}=4.1$, $\mathrm{dU} / \mathrm{dC}=3.75$, and $5-\mathrm{MedC} / \mathrm{dU}=1.1$.

Osmylated $\mathrm{T}$ is stable at room temperature, but osmylated $\mathrm{C}$ hydrolyzes/deaminates slowly to form dU [52]. Under our conditions, water at room temperature, the reaction is about 1 to $2 \%$ per hour (unpublished results). Protocol B, as described in [46], to effectively osmylate $\mathrm{dC}$ takes $11 \mathrm{~h}$, so conversion of $\mathrm{C}$ to $U$ would become important, and perhaps mislead the nanopore measurements. We recognized this problem early, and developed a new procedure for OsBp preparation and new protocols [53] that we applied to the preparation of the osmylated oligos in this study. The new protocols are summarized below. The new OsBp preparation is still $15.75 \mathrm{mM}$ in $\mathrm{OsO}_{4}$, just as in the old protocols $[42,46]$, but prepared in saturated 2,2'-bipyridine using a 4 to 8 -fold molar excess of the later. After vigorous mixing of the two components, the supernatant is removed and used as the new stock solution (OsBp $15.75 \mathrm{mM}$ in saturated 2,2'-bipyridine (sat. bipy)). Saturated 2,2'-bipyridine in water is approximately $30 \mathrm{mM}$ as indicated in the literature. Experiments and kinetic determinations with the new OsBp stock solution (unpublished results) revealed that the reactivity is 4-fold higher compared to the OsBp 1:1 preparation, as described in $[42,46]$. Hence, we recommend the new protocol $\mathrm{A}$ as $60 \mathrm{~min}$ incubation in $1.575 \mathrm{mM}$ OsBp (sat. bipy), and the new protocol B as $110 \mathrm{~min}$ incubation in $12.6 \mathrm{mM}$ OsBp (sat. bipy). Please note that the stock solution is saturated in bipyridine, because of the way it was prepared. However the result- ing reaction mixtures, because they are accordingly diluted (either to $1.575 \mathrm{mM}$ or to $12.6 \mathrm{mM}$ ), are no longer saturated in bipyridine. Based on the new reactivities (unpublished results) new protocol A results in approximately 95\% T-osmylation and 10\% C-osmylation; whereas new protocol $\mathrm{B}$ results in over $99.99 \%$ T-osmylation and $99.99 \%$ C-osmylation. Care was taken that $\mathrm{C}$-osmylated oligos were kept refrigerated/ frozen.

HPLC was conducted with an Agilent 1100/1200 HPLC equipped with a binary pump and individual thermostats for autosampler and column compartment. Chemstation software Rev.B.04.01 SP1 were used for data acquisition and processing. The column was a $2 \times 250 \mathrm{~mm}$ BioLC DNAPac ${ }^{\circledR}$ PA200 from Dionex used in conjunction with $25 \mathrm{mM}$ Tris $\cdot \mathrm{HCl} \mathrm{pH} 7$ buffer and a $\mathrm{NaCl}$ gradient; the column was maintained at $30^{\circ} \mathrm{C}$. This type of ion-exchange chromatography resolves oligos based on length and composition.

Part B, Chemicals, materials and instrumentation for nanopore measurements: Nanopore experiments were conducted with $10 \mu \mathrm{M}$ DNA in $1.0 \mathrm{M} \mathrm{KCl}, 10 \mathrm{mM}$ potassium phosphate buffer at $\mathrm{pH} 7.4$ and at $22 \pm 1{ }^{\circ} \mathrm{C}$, as described in detail in [14], and summarized here. WT $\alpha$-hemolysin was purchased from List Biological Laboratories in the monomer form of lyophilized power and dissolved in water at $1 \mathrm{mg} / \mathrm{mL}$. 1,2Diphytanoyl-sn-glycero-3-phosphocholine (DPhPC) was dissolved in decane at $10 \mathrm{mg} / \mathrm{mL}$ and used to form the bilayer. The bilayer was supported by a glass nanopore membrane (GNM), which was modified with a $2 \%(\mathrm{v} / \mathrm{v})$ (3-cyanopropyl)dimethylchlorosilane in acetonitrile to create a moderately hydrophobic surface. Current-time $(I-t)$ recordings were performed at $22 \pm 1{ }^{\circ} \mathrm{C}$ using a custom-built high impedance and low-noise system (Electronic BioSciences Inc., San Diego, CA) for the Chemistry Department, University of Utah. The $\mathrm{KCl}$ solution was used as the electrolyte to fill the solution reservoir and the GNM capillary. A voltage was applied across the GNM between two $\mathrm{Ag} / \mathrm{AgCl}$ electrodes placed inside and outside of the capillary. A lipid bilayer was deposited across the GNM orifice as indicated by a resistance increase from ca. $10 \mathrm{M} \Omega$ (associated with the open GNM) to ca. $100 \mathrm{G} \Omega$. A pressure of 20 to $40 \mathrm{mmHg}$ was applied to the inside of the GNM capillary using a syringe, allowing the lipid bilayer to be functional for the protein channel reconstitution. Next, $0.2 \mu \mathrm{L}$ of $\alpha$-HL monomer solution at $1 \mathrm{mg} / \mathrm{mL}$ was added to the cis side of GNM (a volume of $350 \mu \mathrm{L}$ ). A voltage of $120 \mathrm{mV}$ (trans vs cis) was applied. The $I-t$ traces were filtered at $100 \mathrm{kHz}$ and sampled at $500 \mathrm{kHz}$. Events were extracted using QuB (version 1.5.0.31), and histograms were analyzed by Origin 9.1. Heat plots were plotted using data analysis programs provided by Electronic BioSciences Inc., San Diego, CA. 


\section{Competing interest statement}

Anastassia Kanavarioti is the Founder and Director of Yenos Analytical LLC, a company engaged in the development and manufacturing of osmylated nucleic acids.

\section{Acknowledgements}

We thank Dr. Cynthia Burrows and Dr. Aaron Fleming (University of Utah), as well as Dr. Geoffrey Barrall (Electronic Biosciences, San Diego, CA) for helpful discussions. Y. D. thanks the NIH for support via R01 GM093099 (to C. B.). The work at Yenos Analytical LLC was supported with private funding.

\section{References}

1. Graham, M. D. JALA (1998-2010) 2003, 8, 72-81. doi:10.1016/S1535-5535(03)00023-6

2. Bayley, H.; Martin, C. R. Chem. Rev. 2000, 100, 2575-2594. doi:10.1021/cr980099g

3. Song, L.; Hobaugh, M. R.; Shustak, C.; Cheley, S.; Bayley, H.; Gouaux, J. E. Science 1996, 274, 1859-1866. doi:10.1126/science.274.5294.1859

4. Faller, M.; Niederweis, M.; Schulz, G. E. Science 2004, 303, 1189-1192. doi:10.1126/science.1094114

5. Simpson, A. A.; Tao, Y.; Leiman, P. G.; Badasso, M. O.; He, Y.; Jardine, P. J.; Olson, N. H.; Morais, M. C.; Grimes, S.; Anderson, D. L.; Baker, T. S.; Rossmann, M. G. Nature 2000, 408, 745-750. doi:10.1038/35047129

6. Li, J.; Stein, D.; McMullan, G.; Branton, D.; Aziz, M. J.; Golovchenko, J. A. Nature 2001, 412, 166-169. doi:10.1038/35084037

7. Kwok, H.; Briggs, K.; Tabard-Cossa, V. PLoS One 2014, 9, e92880. doi:10.1371/journal.pone.0092880

8. Garaj, S.; Liu, S.; Golovchenko, J. A.; Branton, D. Proc. Natl. Acad. Sci. U. S. A. 2013, 110, 12192-12196. doi:10.1073/pnas.1220012110

9. Kudr, J.; Skalickova, S.; Nejdl, L.; Moulick, A.; Ruttkay-Nedecky, B.; Adam, V.; Kizek, R. Electrophoresis 2015, 36, 2367-2379. doi:10.1002/elps.201400612

10. Kasianowicz, J. J. Chem. Rev. 2012, 112, 6215-6217. doi:10.1021/cr300444k

11. Reiner, J. E.; Balijepalli, A.; Robertson, J. W. F.; Campbell, J.; Suehle, J.; Kasianowicz, J. J. Chem. Rev. 2012, 112, 6431-6451. doi:10.1021/cr300381m

12. Haque, F.; Li, J.; Wu, H.-C.; Liang, X.-J.; Guo, P. Nano Today 2013, 8 , 56-74. doi:10.1016/j.nantod.2012.12.008

13. Maglia, G.; Heron, A. J.; Stoddart, D.; Japrung, D.; Bayley, H. Methods Enzymol. 2010, 475, 591-623. doi:10.1016/S0076-6879(10)75022-9

14. Jin, Q.; Fleming, A. M.; Johnson, R. P.; Ding, Y.; Burrows, C. J.; White, H. S. J. Am. Chem. Soc. 2013, 135, 19347-19353. doi:10.1021/ja410615d

15. Stoddart, D.; Heron, A. J.; Mikhailova, E.; Maglia, G.; Bayley, H. Proc. Natl. Acad. Sci. U. S. A. 2009, 106, 7702-7707. doi:10.1073/pnas.0901054106

16. Wolna, A. H.; Fleming, A. M.; An, N.; He, L.; White, H. S.; Burrows, C. J. Isr. J. Chem. 2013, 53, 417-430. doi:10.1002/ijch.201300022
17. An, N.; Fleming, A. M.; White, H. S.; Burrows, C. J. Proc. Natl. Acad. Sci. U. S. A. 2012, 109, 11504-11509. doi:10.1073/pnas.1201669109

18. Ding, Y.; Fleming, A. M.; He, L.; Burrows, C. J. J. Am. Chem. Soc. 2015, 137, 9053-9060. doi:10.1021/jacs.5b03912

19. Kasianowicz, J. J.; Brandin, E.; Branton, D.; Deamer, D. W. Proc. Natl. Acad. Sci. U. S. A. 1996, 93, 13770-13773. doi:10.1073/pnas.93.24.13770

20. Akeson, M.; Branton, D.; Kasianowicz, J. J.; Brandin, E.; Deamer, D. W. Biophys. J. 1999, 77, 3227-3233. doi:10.1016/S0006-3495(99)77153-5

21. Meller, A.; Nivon, L.; Brandin, E.; Golovchenko, J.; Branton, D. Proc. Natl. Acad. Sci. U. S. A. 2000, 97, 1079-1084. doi:10.1073/pnas.97.3.1079

22. National Institute of Health. THE $\$ 1000$ GENOME. http://grants.nih.gov/grants/guide/rfa-files/RFA-HG-04-003.html (accessed Jan 11, 2016). http://www.genome.gov/10000368.

23. Branton, D.; Deamer, D. W.; Marziali, A.; Bayley, H.; Benner, S. A.; Butler, T.; Di Ventra, M.; Garaj, S.; Hibbs, A.; Huang, X.; Jovanovich, S. B.; Krstic, P. S.; Lindsay, S.; Ling, X. S.; Mastrangelo, C. H.; Meller, A.; Oliver, J. S.; Pershin, Y. V.; Ramsey, J. M.; Riehn, R.; Soni, G. V.; Tabard-Cossa, V.; Wanunu, M.; Wiggin, M.; Schloss, J. A. Nat. Biotechnol. 2008, 26, 1146-1153. doi:10.1038/nbt.1495

24. Bayley, H. Clin. Chem. 2015, 61, 25-31. doi:10.1373/clinchem.2014.223016

25. Wanunu, M. Phys. Life Rev. 2012, 9, 125-158. doi:10.1016/j.plrev.2012.05.010

26. Feng, Y.; Zhang, Y.; Ying, C.; Wang, D.; Du, C. Genomics, Proteomics Bioinf. 2015, 13, 4-16. doi:10.1016/j.gpb.2015.01.009

27. Venkatesan, B. M.; Bashir, R. Nat. Nanotechnol. 2011, 6, 615-624. doi:10.1038/nnano.2011.129

28. Ashkenasy, N.; Sánchez-Quesada, J.; Bayley, H.; Ghadiri, M. R. Angew. Chem., Int. Ed. 2005, 44, 1401-1404. doi:10.1002/anie.200462114

29. Stoddart, D.; Heron, A. J.; Klingelhoefer, J.; Mikhailova, E.; Maglia, G.; Bayley, H. Nano Lett. 2010, 10, 3633-3637. doi:10.1021/nl101955a

30. Butler, T. Z.; Pavlenok, M.; Derrington, I. M.; Niederweis, M.; Gundlach, J. H. Proc. Natl. Acad. Sci. U. S. A. 2008, 105 , 20647-20652. doi:10.1073/pnas.0807514106

31. Stoddart, D.; Ayub, M.; Höfler, L.; Raychaudhuri, P.; Klingelhoefer, J. W.; Maglia, G.; Heron, A.; Bayley, H. Proc. Natl. Acad. Sci. U. S. A. 2014, 111, 2425-2430. doi:10.1073/pnas.1312976111

32. Ervin, E. N.; Barrall, G. A.; Pal, P.; Bean, M. K.; Schibel, A. E. P.; Hibbs, A. D. Bionanosci. 2014, 4, 78-84. doi:10.1007/s12668-013-0119-0

33. Mitchell, N.; Howorka, S. Angew. Chem., Int. Ed. 2008, 47, 5565-5568. doi:10.1002/anie.200800183

34. Kumar, S.; Tao, C.; Chien, M.; Hellner, B.; Balijepalli, A.; Robertson, J. W.; Li, Z.; Russo, J. J.; Reiner, J. E.; Kasianowicz, J. J.; Ju, J. Sci. Rep. 2012, 2, No. 684. doi:10.1038/srep00684

35. Borsenberger, V.; Mitchell, N.; Howorka, S. J. Am. Chem. Soc. 2009, 131, 7530-7531. doi:10.1021/ja902004s

36. Laszlo, A. H.; Derrington, I. M.; Ross, B. C.; Brinkerhoff, H.; Adey, A.; Nova, I. C.; Craig, J. M.; Langford, K. W.; Samson, J. M.; Daza, R.; Doering, K.; Shendure, J.; Gundlach, J. H. Nat. Biotechnol. 2014, 32, 829-833. doi:10.1038/nbt.2950 
37. Loman, N. J.; Quinlan, A. R. Bioinformatics 2014, 30, 3399-3401. doi:10.1093/bioinformatics/btu555

38. Jain, M.; Fiddes, I. T.; Miga, K. H.; Olsen, H. E.; Paten, B.; Akeson, M. Nat. Methods 2015, 12, 351-356. doi:10.1038/nmeth.3290

39. Chu, J.; González-López, M.; Cockroft, S. L.; Amorin, M.; Ghadiri, M. R. Angew. Chem., Int. Ed. 2010, 49, 10106-10109. doi:10.1002/anie.201005460

40. Lieberman, K. R.; Cherf, G. M.; Doody, M. J.; Olasagasti, F.; Kolodji, Y.; Akeson, M. J. Am. Chem. Soc. 2010, 132, 17961-17972. doi:10.1021/ja1087612

41. Manrao, E. A.; Derrington, I. M.; Laszlo, A. H.; Langford, K. W.; Hopper, M. K.; Gillgren, N.; Pavlenok, M.; Niederweis, M.; Gundlach, J. H. Nat. Biotechnol. 2012, 30, 349-353. doi:10.1038/nbt.2171

42. Kanavarioti, A.; Greenman, K. L.; Hamalainen, M.; Jain, A.; Johns, A. M.; Melville, C. R.; Kemmish, K.; Andregg, W. Electrophoresis 2012, 33, 3529-3543. doi:10.1002/elps.201200214

43. Beer, M.; Stern, S.; Carmalt, D.; Mohlhenrich, K. H. Biochemistry 1966, 5, 2283-2288. doi:10.1021/bi00871a017

44. Chang, C.-H.; Beer, M.; Marzilli, L. G. Biochemistry 1977, 16, 33-38. doi:10.1021/bi00620a006

45. Paleček, E. Methods Enzymol. 1992, 212, 139-155. doi:10.1016/0076-6879(92)12010-N

46. Kanavarioti, A. Nanotechnology 2015, 26, 134003. doi:10.1088/0957-4484/26/13/134003

47. Kanavarioti, A. Austin J. Proteomics Bioinf. Genomics 2015, 2, 1012.

48. Osmylation is used in the literature to describe the reaction of $\mathrm{OsO}_{4}$. For simplicity we use the same term here to describe the reaction of the complex osmium tetroxide 2,2'-bipyridine (OsBp). Hence osmylated oligos/DNA are the products carrying the OsBp moiety.

49. Liang, X.; Kuhn, H.; Frank-Kamenetskii, M. D. Biophys. J. 2006, 90 , 2877-2889. doi:10.1529/biophysj.105.074104

50. Henley, R. Y.; Vazquez-Pagan, A. G.; Johnson, M.; Kanavarioti, A.; Wanunu, M. PLoS One 2015, 10, e0142155. doi:10.1371/journal.pone.0142155

51. Mathé, J.; Aksimentiev, A.; Nelson, D. R.; Schulten, K.; Meller, A. Proc. Natl. Acad. Sci. U. S. A. 2005, 102, 12377-12382. doi:10.1073/pnas.0502947102

52. Chang, C.-H.; Ford, H.; Behrman, E. J. Inorg. Chim. Acta 1981, 55, 77-80. doi:10.1016/S0020-1693(00)90785-0

53. Kanavarioti, A. US application \#14/944,888, patent pending.

\section{License and Terms}

This is an Open Access article under the terms of the Creative Commons Attribution License

(http://creativecommons.org/licenses/by/2.0), which permits unrestricted use, distribution, and reproduction in any medium, provided the original work is properly cited.

The license is subject to the Beilstein Journal of Nanotechnology terms and conditions:

(http://www.beilstein-journals.org/bjnano)

The definitive version of this article is the electronic one which can be found at:

doi:10.3762/bjnano.7.11 